\title{
SPINOR FRENET EQUATIONS IN THREE DIMENSIONAL LIE GROUPS
}

\author{
O. ZEKI OKUYUCU, Ö. GÖKMEN YILDIZ, AND MURAT TOSUN \\ Abstract. In this paper, we study spinor Frenet equations in three dimen- \\ sional Lie Groups with a bi-invariant metric. Also, we obtain spinor Frenet \\ equations for special cases of three dimensional Lie groups.
}

\section{INTRODUCTION}

In differential geometry, we think that curves are geometric set of points of loci. Curves theory is a important workframe in the differential geometry studies. Geometric properties of a curve are heavily studied for a long time and are still studied in Euclidean space and other spaces. One of the most important tools used to analyze a curve is the Frenet frame, a moving frame that provides an orthonormal coordinate system at each point of the curve. We can show that this orthonormal system with $\{T, N, B\}$ in Euclidean 3-space, which are called tangent vector field, principal normal vector field and binormal vector field, respectively. And for a curve, we can calculate curvature and torsion along the curve with the help of Frenet vectors. They are so useful for characterizations of special curves like that general helices, Slant helices, Mannheim curves, Bertrand curves etc. Characterizations of these curves are studied in different spaces and we can

2010 Mathematics Subject Classification. Primary 11A66; Secondary 22E15.

Key words and phrases. Spinor, Frenet equations, Lie groups. 
see the applications of special curves in nature, mechanic tools, computer aided design and computer graphics etc.

Recently, Castillo and Barrales studied spinor formulation of the Frenet equations of the curve and they obtained spinor equivalent of the Frenet equations for

a curve [5]. Moreover Kişi et.al. and Ünal et. al. defined spinor formulations of the Frenet equations of a curve according to Darboux Frame and Bishop Frame, respectively (see [6] and [7]).

In this paper, we define spinor formulation of the Frenet equations of the curve in three dimensional Lie Groups. Also we give this formulation for special cases of three dimensional Lie groups. We believe that this study will be useful for curves theory in Lie groups.

\section{Preliminaries}

Let $G$ be a Lie group with a bi-invariant metric $\langle$,$\rangle and D$ be the Levi-Civita connection of Lie group $G$. If $\mathfrak{g}$ denotes the Lie algebra of $G$ then we know that $\mathfrak{g}$ is issomorphic to $T_{e} G$ where $e$ is neutral element of $G$. If $\langle$,$\rangle is a bi-invariant$ metric on $G$ then we have

$$
\langle X,[Y, Z]\rangle=\langle[X, Y], Z\rangle
$$

and

$$
D_{X} Y=\frac{1}{2}[X, Y]
$$

for all $X, Y$ and $Z \in \mathfrak{g}$.

Let $\alpha: I \subset \mathbb{R} \rightarrow G$ be an arc-lenghted curve and $\left\{X_{1}, X_{2}, \ldots, X_{n}\right\}$ be an orthonormal basis of $\mathfrak{g}$. In this case, we write that any two vector fields $W$ and $Z$ 
along the curve $\alpha$ as $W=\sum_{i=1}^{n} w_{i} X_{i}$ and $Z=\sum_{i=1}^{n} z_{i} X_{i}$ where $w_{i}: I \rightarrow \mathbb{R}$ and $z_{i}: I \rightarrow \mathbb{R}$ are smooth functions. Also the Lie bracket of two vector fields $W$ and $Z$ is given

$$
[W, Z]=\sum_{i=1}^{n} w_{i} z_{i}\left[X_{i}, X_{j}\right]
$$

and the covariant derivative of $W$ along the curve $\alpha$ with the notation $D_{\alpha^{\prime}} W$ is given as follows

$$
D_{\alpha^{\prime}} W=\dot{W}+\frac{1}{2}[T, W]
$$

where $T=\alpha^{\prime}$ and $\dot{W}=\sum_{i=1}^{n} \dot{w}_{i} X_{i}$ or $\dot{W}=\sum_{i=1}^{n} \frac{d w}{d t} X_{i}$. Note that if $W$ is the left-invariant vector field to the curve $\alpha$ then $\dot{W}=0$ (see [3] for details).

Let $G$ be a three dimensional Lie group and $(T, N, B, \varkappa, \tau)$ denote the Frenet apparatus of the curve $\alpha$, and calculate $\varkappa=\|T\|$.

Definition 2.1. Let $\alpha: I \subset \mathbb{R} \rightarrow G$ be a parametrized curve with the Frenet apparatus $(T, N, B, \varkappa, \tau)$ then

$$
\tau_{G}=\frac{1}{2}\langle[T, N], B\rangle
$$

or

$$
\tau_{G}=\frac{1}{2 \varkappa^{2} \tau}\langle\ddot{T},[T, \dot{T}]\rangle+\frac{1}{4 \varkappa^{2} \tau}\|[T, \dot{T}]\|^{2}
$$

(see [2]). 
Proposition 2.2. Let $\alpha: I \subset \mathbb{R} \rightarrow G$ be an arc length parametrized curve with the Frenet apparatus $\{T, N, B\}$. Then the following equalities

$$
\begin{aligned}
& {[T, N]=\langle[T, N], B\rangle B=2 \tau_{G} B} \\
& {[T, B]=\langle[T, B], N\rangle N=-2 \tau_{G} N}
\end{aligned}
$$

hold (see [8]).

In the following sentences we give some properties about spinors:

We can represent a spinor

$$
\phi=\left(\begin{array}{l}
\phi_{1} \\
\phi_{2}
\end{array}\right)
$$

by way of three vector $a, b, c \in \mathbb{R}^{3}$ so that

$$
a+i b=\phi^{t} \sigma \phi, \quad c=-\widehat{\phi}^{t} \sigma \phi,
$$

where the superscript $t$ denotes transposition and $\widehat{\phi}$ is the mate (or conjugate [1])

$$
\widehat{\phi} \equiv-\left(\begin{array}{cc}
0 & 1 \\
-1 & 0
\end{array}\right), \bar{\phi}=\left(\begin{array}{c}
-\bar{\phi}_{2} \\
\bar{\phi}_{1}
\end{array}\right)
$$

where the bar indicates complex conjugation. In addition, $\sigma=\left(\sigma_{1}, \sigma_{2}, \sigma_{3}\right)$ is a vector whose cartesian components are the complex symmetrics $2 \times 2$ matrices

$$
\sigma_{1}=\left(\begin{array}{cc}
1 & 0 \\
0 & -1
\end{array}\right), \sigma_{2}=\left(\begin{array}{cc}
i & 0 \\
0 & i
\end{array}\right), \sigma_{3}=\left(\begin{array}{cc}
0 & -1 \\
-1 & 0
\end{array}\right) .
$$


Then, the vector $a, b$ and $c$ are clearly given by

$$
\begin{aligned}
a+i b & =\left(\phi_{1}^{2}-\phi_{2}^{2}, i\left(\phi_{1}^{2}+\phi_{2}^{2}\right),-2 \phi_{1} \phi_{2}\right) \\
c & =\left(\phi_{1} \bar{\phi}_{2}+\bar{\phi}_{1} \phi_{2}, i\left(\bar{\phi}_{2} \phi_{1}-\bar{\phi}_{1} \phi_{2}\right),\left|\phi_{1}\right|^{2}-\left|\phi_{2}\right|^{2}\right)
\end{aligned}
$$

Since the vector $a+i b$ is a isotropic vector, by way of an explict computation one finds that $a, b$ and $c$ are one another orthogonal and $|a|=|b|=|c|=\bar{\phi}^{t} \phi$. Moreover $\langle a \times b, c\rangle=\operatorname{det}(a, b, c)>0$.

Conversely, if the vector $a, b$ and $c$ one another orthogonal vectors of same magnitude $(\langle a \times b, c\rangle>0)$, then there is a spinor defined up to sign such that the Eq. (2.5) holds.

As it is mentioned the above, for two arbitrary $\psi$ and $\phi$, there exist following equalities

$$
\begin{aligned}
\overline{\psi^{t} \sigma \phi} & =-\widehat{\psi^{t}} \sigma \widehat{\phi} \\
\widehat{a \psi+b} \phi & =\bar{a} \widehat{\psi}+\bar{b} \widehat{\phi} \\
\widehat{\widehat{\phi}} & =-\phi
\end{aligned}
$$

where $a$ and $b$ are complex numbers [1] and [5].

The relations between spinors and orthonormal bases given in Eq. (2.5) is two to on; the spinors $\phi$ and $-\phi$ correspond to the same ordered orthonormal bases $\{a, b, c\}$, with $|a|=|b|=|c|$ and $\langle a \times b, c\rangle>0$. Moreover we can define different spinors by using the ordered triads $\{a, b, c\},\{b, c, a\}$ and $\{c, a, b\}$. 
The equation $\psi^{t} \sigma \phi=\phi^{t} \sigma \psi$ is satisfied for any pair of $\psi$ and $\phi$, because the matrices $\sigma$ given by Eq. (2.6) are symmetric. For any spinor $\phi$ and its conjugate $\widehat{\phi}$ are linearly independent, where $\phi \neq 0$.

\section{Spinor Frenet equations in a three dimensional Lie groups}

In this section we define the spinor Frenet equations for a curve in a three dimensional Lie group $G$ with a bi-invariant metric $\langle$,$\rangle . Also we give the spinor$ Frenet equations in the special cases of $G$.

Let $\alpha: I \subset \mathbb{R} \rightarrow G$ be a curve with arc-length parameter $s$. Then we can wirte with the help of the Eq. (2.3) and Proposition 2.2 the following equations for Frenet vectors of the curve $\alpha$

$$
\left[\begin{array}{c}
\frac{d T}{d s} \\
\frac{d N}{d s} \\
\frac{d B}{d s}
\end{array}\right]=\left[\begin{array}{ccc}
0 & \varkappa & 0 \\
-\varkappa & 0 & \left(\tau-\tau_{G}\right) \\
0 & -\left(\tau-\tau_{G}\right) & 0
\end{array}\right]\left[\begin{array}{c}
T \\
N \\
B
\end{array}\right]
$$

where $\{T, N, B\}$ is Frenet frame, $\tau_{G}=\frac{1}{2}\langle[T, N], B\rangle$ and $\varkappa, \tau$ are curvatures of the curve $\alpha$ in three dimensional Lie group $G$, respectively.

According to the results presented in section 2, there exists a spinor $\phi$, such that

$$
N+i B=\phi^{t} \sigma \phi, \quad T=\widehat{\phi}^{t} \sigma \phi
$$

with $\overline{\phi^{t}} \phi=1$. Therefore the spinor $\phi$ denotes the triad $\{N, B, T\}$. And the Frenet equations for each point of the curve $\alpha$ must correspond to some expression for $\frac{d \phi}{d s}$. 
As $\{\phi, \widehat{\phi}\}$ is a basis for the two component spinors $(\phi \neq 0)$, there are two functions $g$ and $h$, such that

$$
\frac{d \phi}{d s}=g \phi+h \widehat{\phi}
$$

where the functions $g$ and $h$ are possibly complex-valued functions.

Diferentiating the first equation in the Eq. (3.2) with respect to $s$ and by using the Frenet equations, we get

$$
\begin{gathered}
\frac{d N}{d s}+i \frac{d B}{d s}=\frac{d}{d s}\left(\phi^{t} \sigma \phi\right) \\
-\varkappa T-i\left(\tau-\tau_{G}\right)\{N+i B\}=\left(\frac{d \phi}{d s}\right)^{t} \sigma \phi+\phi^{t} \sigma\left(\frac{d \phi}{d s}\right) .
\end{gathered}
$$

If we think together the Eq. (3.3) and Eq. (3.4), using again the Eq. (3.2), we get

$$
-\varkappa T-i\left(\tau-\tau_{G}\right)\{N+i B\}=2 g(N+i B)-2 h(T) .
$$

From the last equation we can write,

$$
g=-i \frac{\left(\tau-\tau_{G}\right)}{2} \text { and } h=\frac{\varkappa}{2}
$$

Thus, we have proved the following theorem.

Theorem 3.1. Let $\alpha$ be an arc-lenghted regular curve with the Frenet vector fields $\{T, N, B\}$ in the Lie group $G$. If its two-component spinors $\phi$ represents the triad $\{N, B, T\}$, then the Frenet equations are equivalent to the single spinor equation

$$
\frac{d \phi}{d s}=-i \frac{\left(\tau-\tau_{G}\right)}{2} \phi+\frac{\varkappa}{2} \widehat{\phi}
$$

where $\tau_{G}=\frac{1}{2}\langle[T, N], B\rangle$ and $\varkappa, \tau$ are curvatures of the curve $\alpha$. 
Now, we introduce this equation for special cases of three dimensional Lie group. In the following remark, we note that three dimensional Lie groups admitting bi-invariant metrics are $S^{3}, S O^{3}$ and Abelian Lie groups using the same notation as in [2] and [4] as follows:

Remark 3.2. Let $G$ be a Lie group with a bi-invariant metric $\langle$,$\rangle . Then the$ following equalities can be given in different Lie groups:

i) If $G$ is abelian group then $\tau_{G}=0$.

ii) If $G$ is $S O^{3}$ then $\tau_{G}=\frac{1}{2}$.

iii) If $G$ is $S^{3}$ then $\tau_{G}=1$

(see for details [2] and [4]).

Corollary 3.3. Let $\alpha$ be an arc-lenghted regular curve with the Frenet vector fields $\{T, N, B\}$ in the Abelian Lie group $G$. If its two-component spinors $\phi$ represents the triad $\{N, B, T\}$, then the Frenet equations are equivalent to the single spinor equation

$$
\frac{d \phi}{d s}=-i \frac{\tau}{2} \phi+\frac{\varkappa}{2} \widehat{\phi}
$$

where $\varkappa$ and $\tau$ are curvatures of the curve $\alpha$.

Proof. If $G$ is Abellian Lie group then using the above Remark and the Theorem 3.1 we have the result.

From the above corollary we can see easily this study is a generalization of the spinor equivalent of the Frenet equations for a curve defined by Del Castillo and 
Barrales [5] in Euclidean 3-space. Moreover, with a similar proof, we have the following two corollaries.

Corollary 3.4. Let $\alpha$ be an arc-lenghted regular curve with the Frenet vector fields $\{T, N, B\}$ in the Lie group $S O^{3}$. If its two-component spinors $\phi$ represents the triad $\{N, B, T\}$, then the Frenet equations are equivalent to the single spinor equation

$$
\frac{d \phi}{d s}=-i \frac{\left(\tau-\frac{1}{2}\right)}{2} \phi+\frac{\varkappa}{2} \widehat{\phi}
$$

where $\varkappa$ and $\tau$ are curvatures of the curve $\alpha$.

Corollary 3.5. Let $\alpha$ be an arc-lenghted regular curve with the Frenet vector fields $\{T, N, B\}$ in the Lie group $S^{3}$. If its two-component spinors $\phi$ represents the triad $\{N, B, T\}$, then the Frenet equations are equivalent to the single spinor equation

$$
\frac{d \phi}{d s}=-i \frac{(\tau-1)}{2} \phi+\frac{\varkappa}{2} \widehat{\phi}
$$

where $\varkappa$ and $\tau$ are curvatures of the curve $\alpha$.

\section{REFERENCES}

[1] E. Cartan, The theory of spinors, Hermann, Paris, 1966. (Dover, New York, reprinted 1981).

[2] Ü. Çiftçi, A generalization of Lancert's theorem, J. Geom. Phys. 59 (2009) 1597-1603.

[3] P. Crouch, F. Silva Leite, The dynamic interpolation problem: on Riemannian manifoldsi Lie groups and symmetric spaces, J. Dyn. Control Syst. 1 (2) (1995) 177-202.

[4] N. do Espírito-Santo, S. Fornari, K. Frensel, J. Ripoll, Constant mean curvature hypersurfaces in a Lie group with a bi-invariant metric, Manuscripta Math. 111 (4) (2003) 459 470. 
[5] T. del Castillo, G. F., Barrales, G.S., Spinor formulation of the differential geometry of curve, Revista Colombiana de Matematicas, Vol 38, (2004), 27-34.

[6] İ., Kişi and M., Tosun, Spinor Darboux Equations of Curves in Euclidean 3-Space, arXiv:1210.4185v1 [math.GM].

[7] D., Ünal, İ., Kişi and M., Tosun. Spinor Bishop Equations of Curves in Euclidean 3-Space, arXiv:1210.0727v1 [math.DG].

[8] O., Z., Okuyucu, I., Gok, Y., Yayl, and N., Ekmekci, Slant Helices in three Dimensional Lie Groups, arXiv:1203.1146v2 [math.DG].

Department of Mathematics, Bilecik Şeyh Edebali University, Turkey

E-mail address: osman.okuyucu@bilecik.edu.tr

Department of Mathematics, Bılecik Şeyh Edebali University, Turkey

E-mail address: ogokmen.yildiz@bilecik.edu.tr

Department of Mathematics, Sakarya University, Turkey

E-mail address: tosun@sakarya.edu.tr 